\title{
Facilitation of Saccadic Eye Movements by Postsaccadic Electrical Stimulation in the Primate Caudate
}

\author{
Kae Nakamura and Okihide Hikosaka \\ Laboratory of Sensorimotor Research, National Eye Institute, National Institutes of Health, Bethesda, Maryland 20892-4435
}

\begin{abstract}
Sensorimotor experience followed by positive feedback leads to motor learning. Although the striatum, an input channel of the basal ganglia, has been implicated to play a key role in motor learning, little is known about how reward information modulates the neuronal processes in the striatum that causes behavioral changes. Here, we report that direct manipulation of the neuronal signal in the primate caudate yields behavioral changes comparable with those induced by natural reward. Electrical stimulation in the oculomotor region of the caudate immediately after saccades to a fixed direction led to selective facilitation of saccades in that direction. The facilitation remained even after stimulation was stopped, indicating a plastic change. These effects were observed when stimulation was applied after, not before, saccades. We propose that the caudate plays a causal role in behavioral changes by integrating selective sensorimotor and reward information in a temporally specific manner.
\end{abstract}

Key words: basal ganglia; caudate; saccade; primate; reinforcement learning; electrical stimulation

\section{Introduction}

The striatum, a part of the basal ganglia, is a key brain structure for sensorimotor learning (Packard and Knowlton, 2002; White and McDonald, 2002; Everitt and Robbins, 2005). Changes in the activity in the striatum is correlated with reinforcement motor learning in animals (Jog et al., 1999; Hassani et al., 2001; Miyachi et al., 2002; Schultz et al., 2003; Barnes et al., 2005; Williams and Eskandar, 2006) and humans (Haruno et al., 2004; O’Doherty et al., 2004; Lehericy et al., 2005; Haruno and Kawato, 2006). The possible cellular mechanisms of reinforcement learning in the striatum have been extensively studied in vitro (Calabresi et al., 1996; Wickens et al., 1996; Partridge et al., 2000; Lovinger et al., 2003; Mahon et al., 2004) and in vivo (Charpier and Deniau, 1997; Charpier et al., 1999; Reynolds and Wickens, 2000, 2002). These studies demonstrate that repetitive excitation of corticostriatal pathways together with dopamine input induce long-term potentiation (LTP) or long-term depression (LTD) of the striatal projection neurons.

Despite these lines of evidence, it is not clear how reward information affects the neuronal processes in the striatum leading to specific behavioral changes. This is attributable in part to the difficulty to pinpointing where and when reward signal is provided, because natural rewards such as liquid or food may affect multiple brain areas over time. One way to address this question is to manipulate neuronal activity locally in the region of interest at a specific time, and then evaluate the resulting behav-

Received Aug. 24, 2006; revised 0ct. 9, 2006; accepted Nov. 2, 2006.

This work was supported by the intramural research program of the National Eye Institute. We thank Drs. Long Ding, Masaki Isoda, Rebecca Berman, Robert Wurtz, and Michele Basso for helpful comments. We also thank GC America, Inc., for providing us with dental acrylic. We also thank the National Institutes of Health fellow's editorial board for editorial assistance.

Correspondence should be addressed to Kae Nakamura, Department of Physiology, University of Wisconsin Madison, \#127 Service Memorial Institute, 1300 University Avenue, Madison, W153706. E-mail: kae@physiology.wisc.edu. DOl:10.1523/JNEUROSCI.3688-06.2006

Copyright $\odot 2006$ Society for Neuroscience $\quad$ 0270-6474/06/2612885-11\$15.00/0 ioral changes. Such an approach has been originally introduced as brain self-stimulation to the medial forebrain bundle (Olds and Milner, 1954). Typically, animals learn to perform a response to receive a brief electrical stimulation to their brains. The significance of these findings is that it allows us to bypass normal physiological input pathways and get direct access to a specific neuronal circuit. The manipulation of the neuronal signal in turn yields behavioral changes, which are comparable with those driven by natural reward (for review, see Olds and Fobes, 1981). In the striatum, positive reinforcement effects have been reported in rats (Wurtz and Olds, 1963; Levine et al., 1971), cats (Justesen et al., 1963; White and Hiroi, 1998), and monkeys (Bursten and Delgado, 1958; Plotnik et al., 1972; Phillips et al., 1976). In this study, we applied a similar procedure in the caudate, a part of the striatum, in monkeys. As a behavioral measure, we studied primate saccadic eye movement by taking advantage of its measurable motor output and its relatively well understood neuronal circuitry.

We previously showed that monkeys' saccadic reaction times as well as the activity of neurons in the oculomotor region in the caudate are strongly modulated by the expected amount of liquid reward (Kawagoe et al., 1998; Lauwereyns et al., 2002a,b; Watanabe et al., 2003b). Therefore, we predicted that electrical stimulation in the caudate would produce behavioral and neural effects that are equivalent to those produced by liquid reward. Indeed, we found that stimulation after saccades in a fixed direction induced a sustained facilitation of saccades in that direction, which were comparable with natural reward-induced behavioral changes. Notably, the stimulation did not simply act as a nonspecific "enhancer" of the stimulation site, but promoted a particular sensorimotor process that preceded the stimulation.

\section{Materials and Methods}

We used four hemispheres of two adult female rhesus monkeys (Macaca mulatta; laboratory designations S and L). Both animals had been im- 
planted with scleral search coils for measuring eye position and a post for holding the head. The recording chambers were placed over the frontoparietal cortices. All aspects of the behavioral experiment, including presentation of stimuli, monitoring of eye movements, monitoring of neuronal activity, and delivery of reward and electrical stimulation were under the control of a QNX-based real-time experimentation data acquisition system (REX). Eye position was monitored by means of a scleral search coil system with $1 \mathrm{~ms}$ resolution. Stimuli generated by an active matrix LCD projector (PJ550; ViewSonic, Walnut, CA) were rearprojected on a frontoparallel screen $25 \mathrm{~cm}$ from the monkey's eyes. Electrical stimulation was controlled by Grass Square pulse stimulator (model S88K; Grass-Telefactor, West Warwick, RI). Drops of water or juice were delivered as reward through a spigot under control of a solenoid valve after successful completion of each trial. Magnetic resonance images were obtained to determine the position of the electrode aimed at the caudate. All procedures were approved by the Institute Animal Care and Use Committee and complied with Public Health Service Policy on the humane care and use of laboratory animals.

Mapping of the caudate nucleus. The purpose of the experiment was to examine the effect of electrical stimulation in the caudate on saccadic eye movements. We therefore mapped saccade-related regions in the caudate by single-unit recordings while the animal performed saccade tasks before all stimulation experiments. The anteroposterior extent of the recording sites was from $8 \mathrm{~mm}$ anterior to $8 \mathrm{~mm}$ posterior to the anterior commissure, which corresponded to anterior 10-26 mm (monkey S) and 7-23 mm (monkey L) in Horsley-Clarke coordinates.

To search saccade-related regions, we tested the visually guided saccade task with asymmetric reward schedule (supplemental Fig. 1, available at www.jneurosci.org as supplemental material), because caudate neurons are sensitive to the biased reward condition (Takikawa et al., 2002). In addition, before each stimulation experiment, we required that caudate neurons near the tip of the electrode were task-related. Note that this asymmetric reward schedule was used only for searching saccaderelated regions. Details of the task have been explained previously (Lauwereyns et al., 2002b). Briefly, after the monkey fixated on a central fixation point for $1200 \mathrm{~ms}$, a peripheral target was presented either to the right or left, to which the animal made a saccade. A liquid reward was delivered if the saccade was correct. In one block of experiment consisting of 20-28 trials (10-14 trials for each direction), the amount of reward was always large $(0.4 \mathrm{ml})$ in one direction of the target and small $(0$ or $0.01 \mathrm{ml}$ ) in the other direction. Even for small reward trials, the animal had to make a correct saccade; otherwise, the same trial was repeated until the saccade was made correctly. In the next block, the direction of reward bias was reversed. These two kinds of blocks with opposite reward schedules were alternated two or three times. Task-related activity, including visual, presaccadic, and pretarget anticipatory activity, were found mainly in the body of the caudate nucleus, $0-6 \mathrm{~mm}$ posterior to the anterior commissure and $2-3 \mathrm{~mm}$ deeper than the upper edge of the caudate nucleus in accordance with previous reports (Hikosaka et al., 1989b,c, 1993). As reported previously, many of these activities were strongly modulated by the reward condition (i.e., large or small reward) (Kawagoe et al., 1998; Watanabe et al., 2003b) or by the rewarded position (Lauwereyns et al., 2002b; Takikawa et al., 2002). Electrical stimulation was applied mainly in these areas. We performed only one experiment at any particular stimulation site, so that each site corresponded to a unique location within the caudate. To see the extent of the effect of electrical stimulation, we tested six series of electrical stimulations at two to four different depths along with the same electrode tracks; the distance between stimulation sites ranged from 1 to $4 \mathrm{~mm}$. The data obtained from the experiments performed outside the caudate task-related regions were not included in the population analyses.

Behavioral task and electrical stimulation. One experiment consisted of three sessions (Fig. 1A). In each session, the animal performed typically 100 trials of visually guided saccades to the left or right (Fig. 1A). After locating an electrode in the task-related region in the caudate and ascertaining task-related neuronal activity, the "prestimulation control" session began (Fig. $1 A$, first row). The monkey performed 100 trials of visually guided saccade to the target $\left(\mathrm{a} 1.2^{\circ} \mathrm{dot}\right)$ presented on either right or left, $20^{\circ}$ from the fixation point. The direction of the target was ran-
A
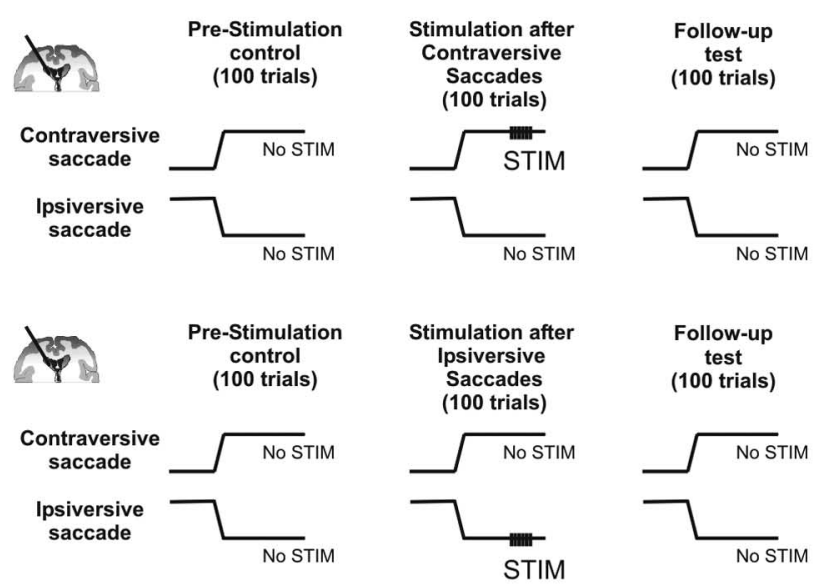

B

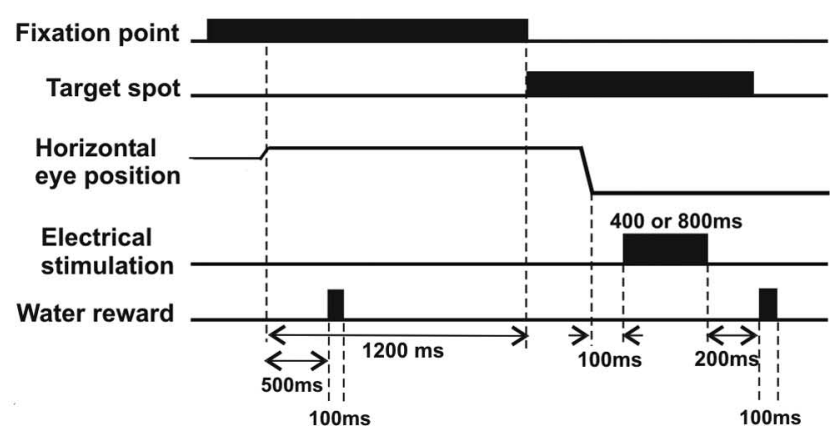

Figure 1. Experimental design. $A$, The schedule of the experiment. In this example, a recording/stimulation electrode is located in the oculomotor region in the left caudate. In each experiment, the monkey performed three sessions of a visually guided saccade task: prestimulation control, stimulation after saccades, and follow-up test. Each session consisted of 100 trials in which the saccade target was presented randomly on the right or left. Only during the "stimulation after saccades" session was electrical stimulation applied to the caudate after saccades in a fixed direction (e.g., to the right or contraversive as shown in the top panel). In another experiment, the stimulation was applied after saccades to the other direction (e.g., to the left or ipsiversive as shown in the bottom panel). In each panel, the schematic horizontal eye position is shown, upward indicating rightward (or contraversive) and downward indicating leftward (or ipsiversive). B, Time events. After the animal fixated on the central fixation point for 1200 $\mathrm{ms}$, a target appeared either in the right or left to which the monkey made a saccade. On a stimulation trial, after the monkey fixated on the target for $100 \mathrm{~ms}$, electrical stimulation was applied for either 400 or $800 \mathrm{~ms}$ (current, up to $50 \mu \mathrm{A}$; pulse duration, $0.5 \mathrm{~ms}$; frequency, 100 $\mathrm{Hz}$ ). A liquid reward ( $0.1 \mathrm{ml}$ ) was given twice per trial: $500 \mathrm{~ms}$ after fixation onset and $200 \mathrm{~ms}$ after the end of the stimulation. On a nonstimulation trial, the animal kept fixating on the target for the same period of time as stimulation period (i.e., 400 or $800 \mathrm{~ms}$ plus $100 \mathrm{~ms}$ ) to obtain the same amount of reward.

domized within a subblock of four trials: two trials for each direction. Thus, one block of 100 trials consisted of 50 saccades to the right and 50 saccades to the left. No electrical stimulation was applied during this session, and this session was used as a control for the following two sessions. Second, in the "stimulation" session, the monkey performed the same visually guided saccade task, but electrical stimulation was applied through the electrode only after correct saccades in a fixed direction, either contraversive or ipsiversive with respect to the location of the electrode. Typically, we applied electrical stimulation after every saccade to the fixed direction (32 experiments for post-contraversive, 39 experiments for post-ipsiversive stimulations). We refer to this procedure as $100 \%$ stimulation schedule. In other experiments (12 for postcontraversive, 7 for post-ipsiversive saccade stimulation), electrical stimulation was applied after $50 \%$ of saccades to the fixed direction associated 
Table 1. Number (percentage) of experiments that showed significant change in the reaction times $(p<0.05$, Mann-Whitney $U$ test)

\begin{tabular}{|c|c|c|c|c|c|c|c|}
\hline \multirow{2}{*}{\multicolumn{2}{|c|}{ Stimulation timing }} & \multicolumn{4}{|c|}{ Presaccade Stim } & & \\
\hline & & \multicolumn{2}{|l|}{ Pretarget } & \multicolumn{2}{|l|}{ Posttarget } & \multicolumn{2}{|c|}{ Postsaccade Stim } \\
\hline \multicolumn{2}{|l|}{ Reaction time change } & Decrease & Increase & Decrease & Increase & Decrease & Increase \\
\hline \multicolumn{8}{|c|}{$\begin{array}{l}\text { 1. Contraversive saccades are associated with stimulation } \\
\text { 1-1. During stimulation }\end{array}$} \\
\hline & & $n=58$ & & $n=15$ & & $n=44$ & \\
\hline Contra Sac & Stim & $15(26 \%)$ & $9(16 \%)$ & $8(53 \%)$ & $4(27 \%)$ & $16(36 \%)$ & $2(5 \%)$ \\
\hline Contra Sac & No Stim & $3(5 \%)$ & $8(14 \%)$ & $1(7 \%)$ & $5(33 \%)$ & & \\
\hline Ipsi Sac & No Stim & $4(7 \%)$ & $14(24 \%)$ & $0(7 \%)$ & $5(33 \%)$ & $5(11 \%)$ & $10(23 \%)$ \\
\hline \multicolumn{8}{|l|}{ 1-2. Follow up } \\
\hline & & $n=45$ & & $n=15$ & & $n=44$ & \\
\hline Contra Sac & No Stim & $1(2 \%)$ & $12(27 \%)$ & $1(7 \%)$ & $5(33 \%)$ & $12(27 \%)$ & $1(2 \%)$ \\
\hline Ipsi Sac & No Stim & $4(9 \%)$ & $0(0 \%)$ & $0(0 \%)$ & $6(40 \%)$ & $6(14 \%)$ & $10(23 \%)$ \\
\hline \multicolumn{8}{|c|}{$\begin{array}{l}\text { 2. Ipsiversive saccades are associated with stimulation } \\
\text { 2-1. During stimulation }\end{array}$} \\
\hline & & $n=54$ & & $n=15$ & & $n=46$ & \\
\hline Ipsi Sac & Stim & $5(9 \%)$ & $29(54 \%)$ & $2(15 \%)$ & $8(62 \%)$ & $13(28 \%)$ & $3(7 \%)$ \\
\hline Ipsi Sac & No Stim & $8(15 \%)$ & $4(7 \%)$ & $1(8 \%)$ & $0(0 \%)$ & & \\
\hline Contra Sac & No Stim & $5(9 \%)$ & $5(9 \%)$ & $1(8 \%)$ & $4(31 \%)$ & $0(0 \%)$ & $17(37 \%)$ \\
\hline \multicolumn{8}{|l|}{ 2-2. Follow up } \\
\hline & & $n=39$ & & $n=15$ & & $n=46$ & \\
\hline Ipsi Sac & No Stim & $4(10 \%)$ & $4(10 \%)$ & $2(13 \%)$ & $0(0 \%)$ & $9(20 \%)$ & $2(4 \%)$ \\
\hline Contra Sac & No Stim & $4(10 \%)$ & $7(18 \%)$ & $1(7 \%)$ & $2(13 \%)$ & $2(4 \%)$ & $14(30 \%)$ \\
\hline
\end{tabular}

'Stim' and 'No Stim' indicate trials associated with or not associated with stimulation, respectively. 'Contra Sac' and 'Ipsi Sac' indicate saccades toward contralateral or ipsilateral side to the electrode, respectively.

stimulation. We refer to this procedure as $50 \%$ stimulation schedule. There was no significant difference in the reaction time changes between the 100 and $50 \%$ stimulation schedules (after contraversive saccade stimulation, $p=0.83$; after ipsiversive saccade stimulation, $p=0.84$ ). Hence the data obtained with both schedules were analyzed together in Figure 3 (for separate analyses, see Table 1). In the last session, "follow-up test," the monkey performed the same visually guided saccade task (100 trials) without stimulation. The purpose of this session was to examine the persistent effect after stimulation was stopped. Each session typically took $\sim 15 \mathrm{~min}$, and the interval between sessions was $\sim 3-5 \mathrm{~min}$.

Detailed timing of electrical stimulation and other events of a trial are demonstrated in Figure $1 B$. Each trial began with the presentation of a central fixation point $\left(0.5^{\circ}\right)$. After the animal maintained fixation on the spot for $1200 \mathrm{~ms}$, the fixation point turned off, and a target, a white small $\operatorname{dot}\left(1.2^{\circ}\right)$, appeared on either right or left, $20^{\circ}$ from the fixation point, to which the animal made a saccade. On the postsaccade stimulation trials, electrical stimulation was applied after maintaining fixation on the target for $100 \mathrm{~ms}$. We used a train of biphasic pulses (pulse duration: $0.5 \mathrm{~ms}, 100$ $\mathrm{Hz}$ ) for the stimulation based on a previous study (Plotnik et al., 1972). The current intensity was usually $50 \mu \mathrm{A}$. The duration of the train of pulses was either $400 \mathrm{~ms}$ ( $n=11$ for post-contraversive, $n=6$ for post-ipsiversive saccade stimulations) or $800 \mathrm{~ms}(n=33$ for postcontraversive, $n=40$ for post-ipsiversive saccade stimulations). There were no significant differences between 400 and $800 \mathrm{~ms}$ postsaccadic stimulations ( $p=0.71$ for post-contraversive; $p=0.70$ for postipsiversive saccade). Hence the data obtained with both schedules were analyzed together (Fig. 3). Electrical stimulation with $50 \mu \mathrm{A}$ occasionally evoked saccades, as reported in cats (Kitama et al., 1991). In such cases, we reduced the intensity of current to $10-30 \mu \mathrm{A}$ so that the stimulation caused no direct motor effects.

An equal amount of liquid reward $(0.1 \mathrm{ml})$ was given twice (Fig. $1 B)$ on both stimulation and nonstimulation trials. The first was $500 \mathrm{~ms}$ after the monkey attained fixation. The second was after the fixation of the target for the same period of stimulation time (i.e., 400 or $800 \mathrm{~ms}$ ) with an additional delay of $200 \mathrm{~ms}$.

To test whether the effects of caudate stimulation depend on the timing of stimulation relative to the target onset or saccade, we also tested electrical stimulation before the saccade. We used two presaccadic stim- ulation schedules: "pretarget stimulation" and "posttarget stimulation" (Fig. $5 A$ ). In the pretarget stimulation, the stimulation was applied for $400 \mathrm{~ms}$ ( $n=23$ for pre-contraversive; $n=21$ for pre-ipsiversive) or 800 $\mathrm{ms}$ ( $n=35$ for pre-contraversive; $n=33$ for pre-ipsiversive) such that it ended simultaneously with the offset of the fixation point. In the posttarget stimulation, the stimulation started simultaneously with the target onset and ended with the onset of the saccade. In both cases, the stimulation was applied before $50 \%$ of saccades directed to the fixed direction (therefore $25 \%$ of all trials) to address whether the behavioral change was a result of a direct stimulation effect or reflected the formation of associations between saccade and stimulation. The schedule of the presaccadic stimulation experiment was the same as the schedule of the postsaccadic stimulation experiment (Fig. $1 A$ ).

Data analysis. As a behavioral measure, we focused on changes in the saccadic reaction times relative to the median reaction time of the prestimulation control session. The reaction time was measured from the target onset to the saccade onset. Incorrect trials such as no fixation (eye position was not on the fixation point for $1500 \mathrm{~ms}$ after its onset), fixation break (eye position was not maintained within $2^{\circ}$ around the fixation point until target onset), wrong saccade (saccade did not reach the point within $5^{\circ}$ around the target), and slow response (saccade did not start within $500 \mathrm{~ms}$ after target onset) were uncommon and excluded from the analyses.

To examine the changes in the saccade reaction time by caudate stimulation, we normalized the reaction time on a given trial by subtracting the median saccadic reaction time during the prestimulation control session from the given reaction time, which was defined as "reaction time change." For the data obtained in each experiment (as shown in Figs. 1 and 5), the reaction time changes during the stimulation and follow-up sessions were compared with those during prestimulation control by Mann-Whitney $U$ test.

To examine the time course of the reaction time changes, we first divided each session (consisting of 100 trials) into 10 periods (consisting of 5 trials in each direction) and averaged the reaction time changes for each period. We then averaged the mean reaction time changes across experiments (as shown in Fig. $4 A$ ).

To examine the residual effects of single caudate stimulation on the reaction times in the subsequent trials, we classified each trial depending 
on the number of trials since the last trial that was associated with caudate stimulation. We then computed the mean and the SE of the reaction time changes for each class of trials (as shown in Fig. $4 B$ ), and performed a KruskalWallis test with Bonferroni's correction $(p<$ $0.01=0.05 / 5)$ to examine whether the mean reaction time for each class of trials was different from zero.

\section{Results}

\section{Postsaccadic stimulation}

\section{facilitates saccades}

We found that postsaccadic stimulation modulated saccadic reaction times. Figure 2, $A-D$, is representative of one experiment, consisting of three sessions (100 trials for each). After locating an electrode in the saccade-related region in the left caudate, we first obtained data in the prestimulation control session ("Control") (Fig. 2A, black dots; $B$, black line). The animal then performed the identical task in the stimulation session, in which electrical stimulation was applied after all contraversive (rightward) saccades (Fig. $2 A, B$, "Stim"). We found that the reaction times of contraversive saccades decreased significantly (median, $199 \mathrm{~ms}$ for the control, $164 \mathrm{~ms}$ for the stimulation session; $p=$ 0.00007 , Mann-Whitney $U$ test). In contrast, the reaction times of the ipsiversive (leftward) saccades increased significantly (median, $123 \mathrm{~ms}$ for the control, $138 \mathrm{~ms}$ for the stimulation session) (Fig. 2C,D) ( $p=0.001$, Mann-Whitney $U$ test $)$.

Importantly, the effect was not a direct motor effect of stimulation because the stimulation was applied after, not before, the initiation of saccades. Indeed, the shortening in the reaction times of contraversive saccades, which were associated with electrical stimulation, remained in the follow-up test even after stimulation was stopped (in the example shown in Fig. 2, median $156 \mathrm{~ms} ; p=0.00007$ compared with prestimulation control; Mann-Whitney $U$ test) (Fig. $2 A$, blue dots; $B$, blue line). The reaction times of ipsiversive saccades also remained significantly longer during the follow-up test (median, $144 \mathrm{~ms}$; $p=0.02$, Mann-Whitney $U$ test) (Fig. $2 C, D$ ).

We performed a total of 44 postsaccadic stimulation experiments for contraversive saccades [Fig. 3A-D, 100\% schedule: $n=$ 32 (all for monkey S), indicated by black lines and circles; $50 \%$ schedule: $n=12$ ( 3 for monkey S, 9 for monkey L), gray lines and circles]. As shown in Figure 3, $A$ and $B$, the reaction times of contraversive saccades decreased significantly in 16 of these experiments ( 13 of 33 for monkey S, 3 of 11 for monkey L) ( $p<$ 0.05 , Mann-Whitney $U$ test), with an average decrease of $18.8 \mathrm{~ms}$ (range, 9.8-34.7 ms). The reaction times increased in two experiments. We also commonly observed a persistent effect during the follow-up test. Among the 16 experiments that showed sig-
Post-Contraversive Saccade Stimulation

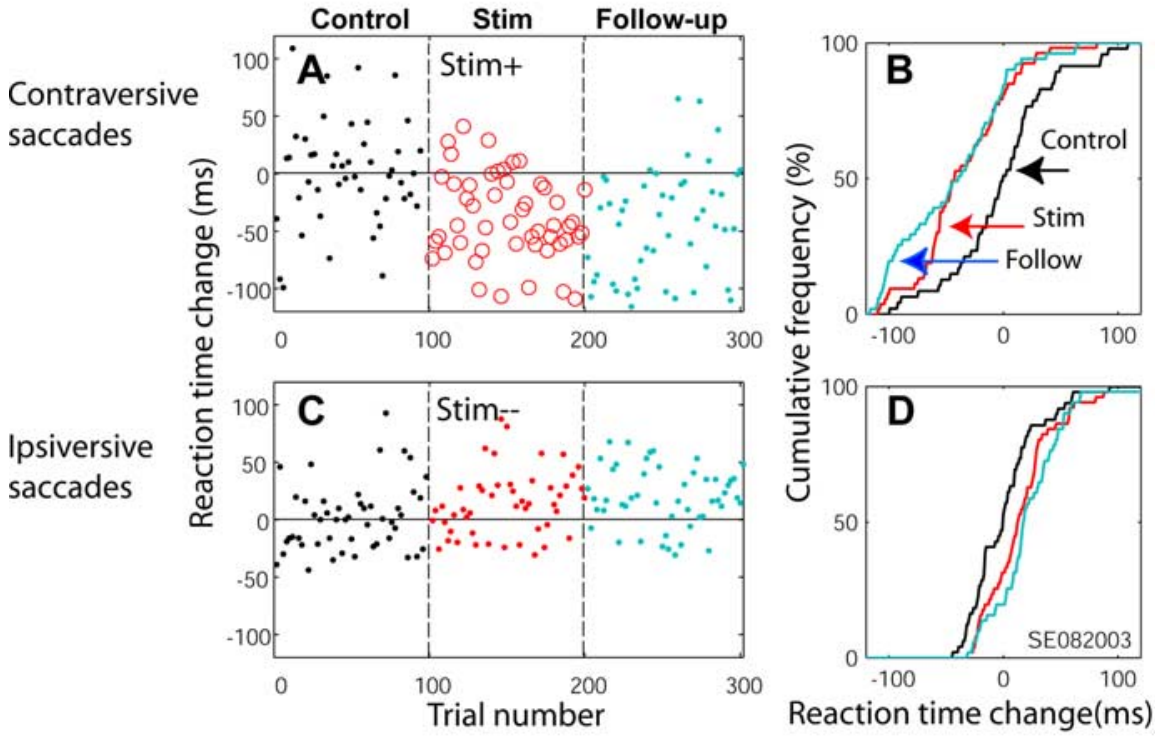

Post-Ipsiversive Saccade Stimulation
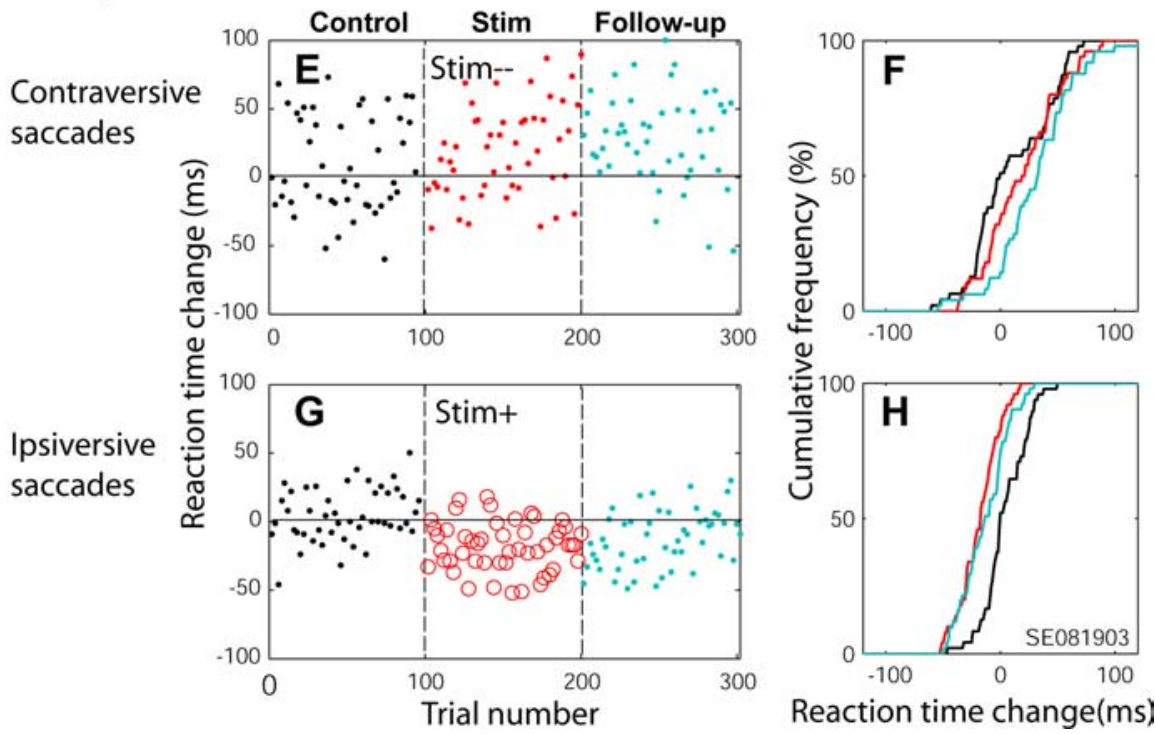

Figure 2. Postsaccadic caudate stimulation facilitates saccades. In these examples, electrical stimulation was applied to the left caudate after all contraversive (rightward) saccades $(\boldsymbol{A}-\boldsymbol{D})$ or all ipsiversive (leftward) saccades $(\boldsymbol{E}-\boldsymbol{H})$. In $\boldsymbol{A}, \boldsymbol{C}, \boldsymbol{E}$, and $\boldsymbol{G}$, the reaction time change of each trial (compared with the median reaction time for the prestimulation control) is plotted against the trial number. The black dots represent the prestimulation control session (Control); red open circles, trials followed by stimulation $($ Stim + ); red dots, trials not followed by stimulation during the stimulation session (Stim-); blue dots, the follow-up test (Follow). $\boldsymbol{B}, \boldsymbol{D}, \boldsymbol{F}$, and $\boldsymbol{H}$ show the cumulative frequency histograms of the reaction time changes. The black, red, and blue lines indicate prestimulation control, stimulation, and follow-up test sessions, respectively.

nificant shortening in the reaction times during the stimulation session, the reaction times remained significantly decreased in 8 experiments during the follow-up test session ( $p<0.05$, MannWhitney $U$ test, compared with prestimulation control). Although shortening in the reaction times tended to be larger in the $100 \%$ schedule (solid circles) than in the 50\% schedule (gray circles), the difference did not reach a significant level ( $p=0.28$, Mann-Whitney $U$ test).

The reaction times of ipsiversive saccades, which were not followed by stimulation, tended to be prolonged, although the effect was less consistent. Of 44 experiments, the reaction times increased in 10 experiments and decreased in 5 experiments (Fig. $3 C, D)$. As observed in contraversive saccades, the prolongation of 


\section{Post-Contraversive Saccade Stimulation}
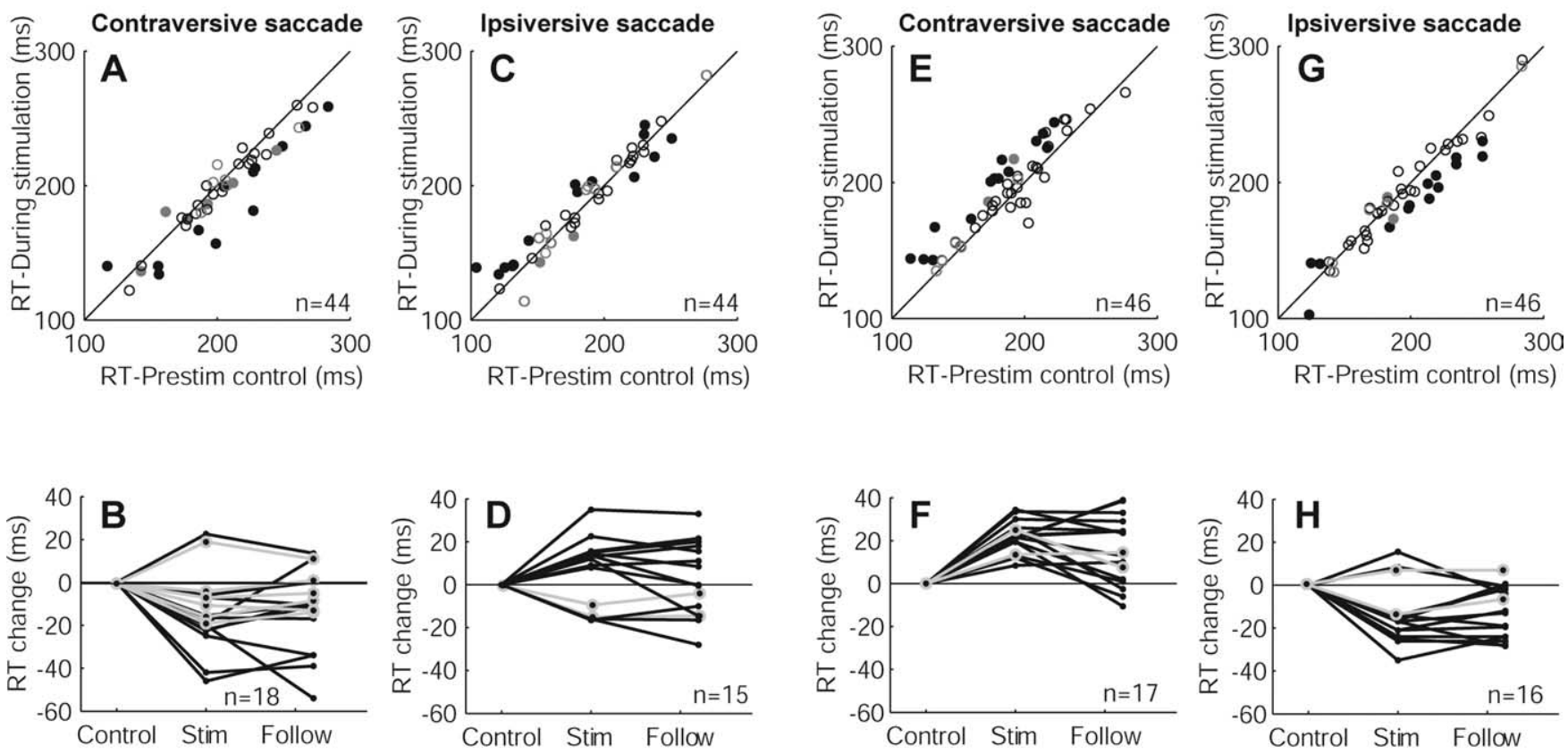

$\multimap$ post-all saccade stimulation

- $\_$post-half saccade stimulation

Figure 3. Population analyses of post-contraversive saccade stimulations $(\boldsymbol{A}-\boldsymbol{D})(n=44$; monkey $\mathrm{S}, n=35$; monkey $\mathrm{L}, n=9)$ and post-ipsiversive saccade stimulations $(\boldsymbol{E}-\boldsymbol{H})(n=46$; monkey $S, n=38$; monkey $\mathrm{L}, n=8$ ). $A$, Median reaction times of contraversive saccades during the stimulation session (ordinate) plotted against the median reaction times during the prestimulation control (abscissa). Each data point corresponds to a single experiment. The filled and open symbols indicate significant and nonsignificant changes, respectively (Mann-Whitney $U$ test, $p<0.05$ ). The black or gray circles/lines indicate the experiments in which all ( $100 \%$ schedule) or one-half ( $50 \%$ schedule) of the trials in the fixed direction of saccades were followed by stimulation, respectively. $\boldsymbol{B}$, Time course of the changes in the median reaction times for contraversive saccades. Control, Prestimulation control session; Stim, stimulation session; Follow, follow-up session. Negative and positive values indicate decrease and increase in the median reaction times compared with the prestimulation control. Data from one experiment are connected by lines. The data from the experiments that showed significant changes in the reaction times (increase or decrease) during the stimulation session are shown. $\boldsymbol{C}, \boldsymbol{D}$, Changes in the median reaction times of ipsiversive saccades for post-contraversive saccade stimulation. The format is the same as in $\boldsymbol{A}$ and $\boldsymbol{B}$. $\boldsymbol{E}-\boldsymbol{H}$, Effects of post-ipsiversive saccades stimulation. The format is the same as in $\boldsymbol{A}-\boldsymbol{D}$.

ipsiversive reaction times tended to persist after stimulation had stopped. Among the 10 experiments that showed significant prolongation in the reaction times during the stimulation session, the reaction times remained significantly prolonged in five experiments during the follow-up test session (Mann-Whitney $U$ test, $p<0.05)$. Unlike facilitation in contraversive saccades, such prolongation in the reaction times for ipsiversive saccades was observed in the $100 \%$ stimulation schedule but not in the $50 \%$ schedule.

\section{The facilitatory effect is selective to the direction of saccades associated with stimulation}

Our findings so far indicate that contraversive eye movements are facilitated when followed by caudate stimulation. To investigate whether this effect is specific to the side of the stimulated caudate or the direction of saccades paired with stimulation, we conducted experiments in which ipsiversive saccades were followed by stimulation (Fig. $1 \mathrm{~A}$, bottom row). In the example shown in Figure 2, $E-H$, the reaction times of ipsiversive saccades were shortened, whereas those of contraversive saccades were prolonged (Fig. $2 E-H$ ). Among a total of 46 experiments (36 for monkey S; 10 for monkey L), the reaction times of ipsiversive saccades became significantly shorter in 13 experiments and longer in 3 experiments ( 10 of 36 for monkey S; 3 of 10 for monkey L) (Fig. 3G). The reaction times of contraversive saccades, which were not associated with stimulation, showed significant prolongation in 17 experiments, whereas none showed a significant shortening (Fig. 3E). These effects tended to remain for both directions of saccades even after the termination of stimulation (Fig. $3 F, H)$. Such a persistent effect did not reach a significant level for the $50 \%$ stimulation schedule $(p=0.42$ for shortening of the reaction times of ipsiversive saccades; $p=0.5$ for prolongation of the reaction times of the contraversive saccades)

These findings indicate that the saccades in a single direction can be facilitated by stimulation of either the ipsilateral or contralateral caudate, so long as the stimulation is fixed to follow saccades in that direction. The changes in saccade reaction times brought about by caudate stimulation are not fixed relative to the side of the caudate being stimulated, but depend on the specific saccade direction that is paired with caudate stimulation.

\section{Time course of the facilitatory effects of}

postsaccadic stimulation

To evaluate further how rapidly caudate stimulation leads to significant changes in behavior, we examined a time course of reaction time changes. The results are shown in Figure $4 \mathrm{~A}$ for the prestimulation control (Control), stimulation (Stim), and follow-up (Follow-up) sessions. Included in this population analysis were 11 experiments in which the reaction times of contraversive saccades were significantly shortened. We found that the facilitation of contraversive saccades by caudate stimulation occurred soon after stimulation was started. The change was significant even after a single trial of stimulation $(p<0.0001)$. 
However, we did not observe additional gradual shortening in reaction times as the cumulative number of stimulations increased (Spearman rank correlation, $\rho=$ $-0.13, p=0.37)$. In contrast, the reaction time change during the follow-up session was slow. Even after stimulation was stopped, the reaction time remained short during the follow-up session. The correlation between the cumulative number of trials and the reaction time did not reach a significant level (Spearman rank correlation, $\rho=0.07, p=0.6$ ). The reaction time change for ipsiversive saccades occurred differently (Fig. $4 A$, bottom row). As the cumulative number of stimulations increased, the reaction time of ipsiversive saccades became gradually longer ( $\rho=$ $0.42, p=0.02)$. The reaction times remained prolonged during the follow-up session.

We also asked how long the facilitatory effect of a single stimulation persisted. Since the target was presented pseudorandomly between the right and left directions and the stimulation was associated with saccades in one direction, a given trial was preceded by a certain number of trials that were not associated with stimulation. In Figure 4, we computed the mean and SE of the saccade reaction time changes against the number of trials since the last trial associated with stimulation. Because of the pseudorandom schedule (i.e., randomized directions within four trials; two for rightward or leftward saccades) (see Materials and Methods), the maximum number of trials since the last stimulation was five in the $100 \%$ stimulation schedule. The reaction times of contraversive saccades, which were associated with stimulation, showed significant shortening up to three trials after the last stimulation applied (Kruskal-Wallis test with Bonferroni's correction, the significance criteria, $p<0.01=0.05 / 5)$. The effect faded away gradually as a function of the number of trials since the last stimulation (Spearman rank correlation, $\rho=0.9, p=0.01$ ).

To summarize, the effect of postsaccadic stimulation is rapid as well as persistent. During the stimulation session, the stimulation facilitated contraversive saccade quickly and the effect decayed gradually. However, once the stimulation is stopped, the effect persisted for a long time.

\section{Facilitatory effect depends on the timing of stimulation}

The results so far indicate that the electrical stimulation in the caudate after saccades to the specific direction facilitates the saccades to that direction. We then asked whether the timing of stimulation relative to the saccade is critical for the effects. To address this issue, in the same saccade-related regions in the caudate of the same animals, we tested the effects of electrical stim-
Post-Contraversive Saccade Stimulation
Control

Control

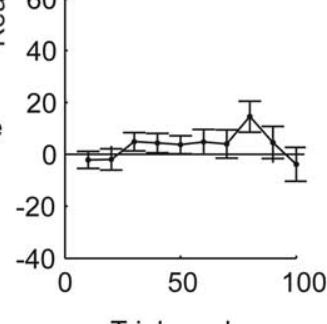

Trial number

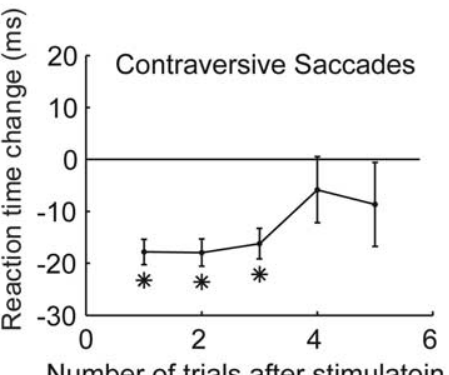

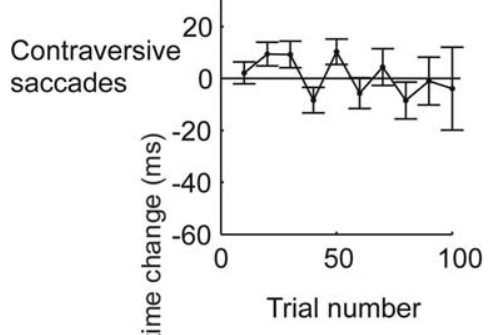

Follow-up
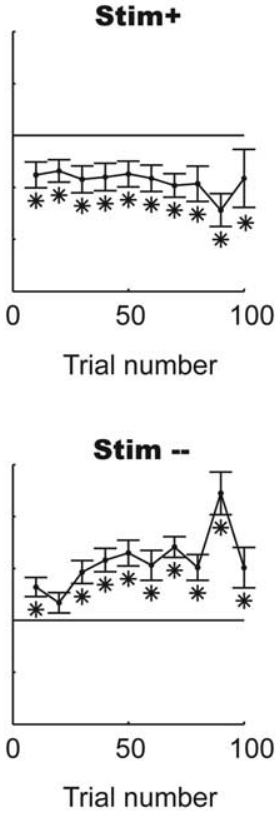

Follow-up

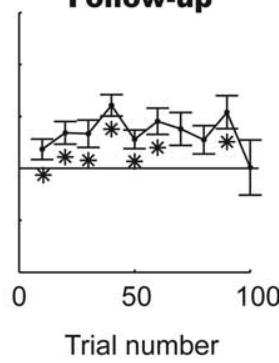

Figure 4. Trial-by-trial changes in the saccade reaction times during the post-contraversive saccade stimulation. $\boldsymbol{A}$, Changes in the reaction times of contraversive saccades (top row) and ipsiversive saccades (bottom row) during control (left column), period), and the reaction time changes (relative to the median reaction time in the control session) were averaged for each period. reaction times during the stimulation session was observed. Note that, during the stimulation session, the reaction times tion session started. This facilitation of saccades remained during the follow-up session. Ipsiversive saccades, which were not ssociated with the stimulation, showed the opposite changes. Asteriskindicates a significant difference from zero (Mann-Whitney Utest, plotted as a function of the number of trials since the last trials associated with caudate stimulation. Note that the facilitatory effect significant difference from zero (Kruskal-Wallis test with Bonferroni's correction, the significance criteria, $p<0.01=0.05 / 5$ ).

ulation applied before saccade generation as separate sets of experiments. We tested two different timings. In the presaccade no. 1 or pretarget stimulation (Fig. $5 \mathrm{~A}$, left), electrical stimulation was applied for $400 \mathrm{~ms}(n=23$ for pre-contraversive; $n=21$ for pre-ipsiversive) or $800 \mathrm{~ms} \mathrm{(} n=35$ for pre-contraversive; $n=33$ for pre-ipsiversive) such that it ended simultaneously with the offset of the fixation point. In the presaccade no. 2 or posttarget stimulation (Fig. 5A, middle), electrical stimulation started at target onset and lasted until saccade onset. The stimulation was given on $50 \%$ of trials for a fixed direction of saccades to address whether the behavioral change was a result of direct stimulation effect or reflected the formation of associations between saccade 
A

Pre-saccade \# (Pre-target) stimulation

Pre-saccade \#2 (Post-target) stimulation
Post-saccade stimulation

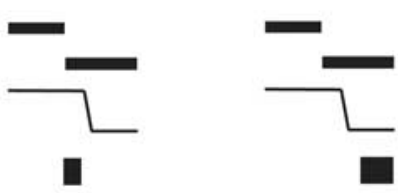

B Pre-Contraversive Saccade Stimulation

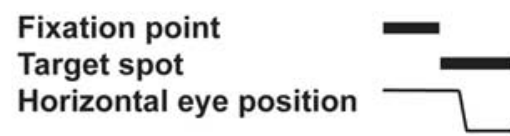

Electrical stimulation

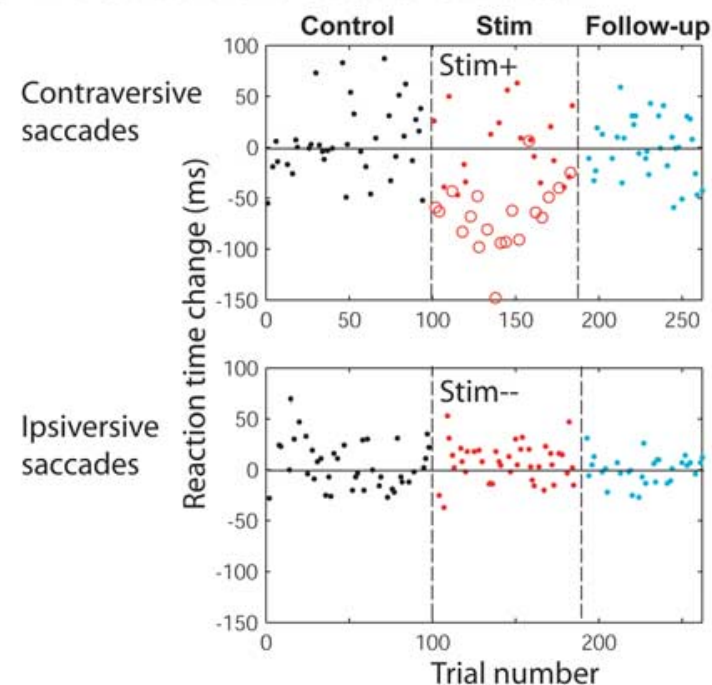

\section{Pre-Ipsiversive Saccade Stimulation}

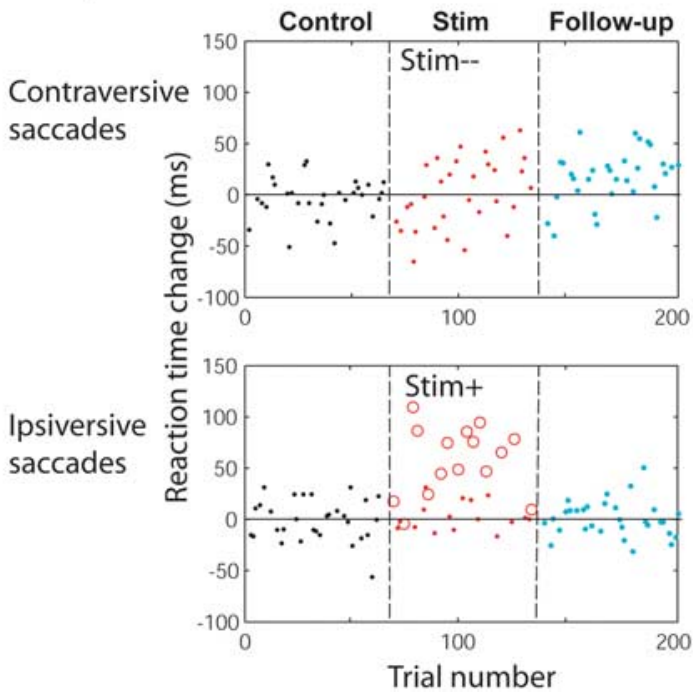

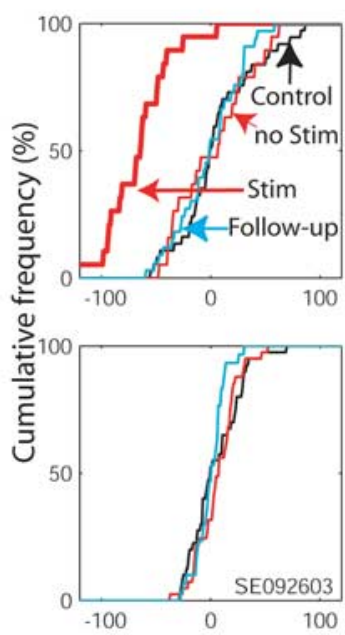

Reaction time change (ms)

times of the contraversive saccades became significantly shorter (Fig. $5 B$, top row, red open circles and thick red line) $(p<$ 0.0001 , Mann-Whitney $U$ test). For the $50 \%$ of contraversive saccades that were not preceded by stimulation, the reaction times did not change significantly (Fig. $5 B$, top row, red dots and thin red lines) $(p=$ $0.84)$. The reaction times of ipsiversive saccades showed no change $(p>0.05$, Mann-Whitney $U$ test). When stimulation was applied before ipsiversive saccades (Fig. $5 C$ ), the reaction times of ipsiversive saccades were significantly prolonged, only when they were preceded by the stimulation $(p<0.0001)$ (Fig. $5 C$, bottom row). The reaction times of contraversive saccades showed no change ( $p>0.05$, Mann-Whitney $U$ test).

Table 1 summarizes the results of all presaccade stimulation experiments. Among 58 pretarget contraversive saccade stimulation experiments (monkey S, $n=$ 38; monkey L, $n=20$ ), a significant decrease in the contraversive saccades was observed in 15 (monkey S, $n=7$; monkey L, $n=8$ ) experiments. Thus, the stimulation before contraversive saccades tended to decrease their reaction times, but it did not reach a significant level $\left(\chi^{2}=0.89\right.$; $\mathrm{df}=2 ; p=0.64)$. In contrast, among 54 pretarget ipsiversive saccade stimulation experiments (monkey S, $n=36$; monkey $\mathrm{L}, n=18$ ), a significant increase in the ipsiversive saccades was observed in 29 (monkey S, $n=24$; monkey L, $n=5$ ) experiments. Thus, the stimulation before ipsiversive saccades significantly increased their reaction times $\left(\chi^{2}=9.8 ; \mathrm{df}=2 ; p=\right.$ 0.0076). Posttarget stimulation showed similar effects. This was different from the results of the postsaccadic stimulation in which the reaction times tended to decrease for the saccades that were associated with the stimulation. In other words, the effects of presaccadic stimulation depended on the side of stimulation relative to the saccades, whereas the effects of postsaccadic stimulation depended on the direction of saccades associated with stimulation.

We also found that presaccadic stimulation induced no significant persistent effect (during the follow-up test). This is shown as an example in Figure 5 for precontraversive saccade stimulation (Fig. $5 B$, top row) $(p=0.66)$ and pre-ipsiversive

Figure 5. Effects of caudate stimulation depend on its timing. $A$, Schematics of three different timings of stimulation. $B, C$ Representative effects of the presaccade no. 1 stimulation, applied before the appearance of a target in the contralateral $(\boldsymbol{B})$ or ipsilateral ( $(\boldsymbol{C})$ side to the electrode. The results are shown in the same format as in Figure 2. During the stimulation session (Stim), the stimulation was applied before $50 \%$ of the contraversive saccades. The red open circles represent trials followed by stimulation; red dots, trials not followed by stimulation. Note that, when preceded by caudate stimulation, the reaction times of contraversive saccades became shortened $(\boldsymbol{B})$, whereas those of ipsiversive saccades became prolonged $(\boldsymbol{C})$. No significant persistent effect was observed during the follow-up test (blue dots and lines).

and stimulation. To have comparable stimulation probability rate, we mainly focused on the comparison between presaccadic stimulation and postsaccadic stimulation with the $50 \%$ schedule.

In the representative of the presaccade no. 1, or pretarget stimulation experiment shown in Figure 5, when stimulation was applied before contraversive saccades (Fig. $5 B$ ), the reaction saccade stimulation (Fig. $5 C$, bottom row) $(p=0.43)$. Similar results were obtained in other presaccadic stimulation experiments (Fig. 6). The reaction times during the follow-up control remained significantly shorter in the postsaccadic stimulation period (Fig. 6C) $(p<0.05)$, but returned to the control level in the presaccadic stimulation (pretarget stimulation: Fig. $6 A, p=$ 
0.77; posttarget stimulation: Fig. $6 B, p=$ 0.29). The lack of persistent effects was also evident by the fact that the reaction times of $50 \%$ of saccades to the direction associated with stimulation, but not preceded by electrical stimulation, did not change significantly during the stimulation block (Fig. 6A, $B$, broken lines, "No stim"). These results suggest that the effects of the pretarget stimulation were attributable to instantaneous changes in saccade preparatory processes, unlike the posttarget stimulation.

\section{Stimulation sites}

Figure $7 A$ shows the locations of taskrelated neurons and stimulation sites in the caudate. To examine whether the effect is localized within the caudate, we performed six series of postsaccadic stimulation experiments at three different depths along the same penetration: the upper and lower borders of the caudate, and the central part of the caudate where task-related neurons were recorded. As shown in Figure $7 B$, shortening in the reaction times was observed when stimulation was applied at the central part of the caudate, but not at the upper or lower border of the caudate. In contrast, stimulation close to task-related neurons (Fig. 7A, open circles) did not always cause significant effects. We could not find a consistent relationship between the types of neuronal activity recorded at the stimulation sites and the presence of stimulation effects. To examine whether the effective stimulation sites tended to be localized within the caudate, we computed the distance of all pairs of stimulation sites that induced significant shortening (Fig. 7A, pink bars), prolongation (cyan bars), or nonsignificant effects (black bars). There was no significant difference in the degree of scattering of stimulation sites of any particular effects ( $p=0.6$, Kruskal-Wallis test).

\section{Discussion}

\section{Postsaccadic stimulation facilitated saccades}

Electrical stimulation in the caudate after saccades in a unique direction induced a short-term and long-lasting facilitation of saccades in that direction. The facilitation of saccades was not a direct motor effect of stimulation because the stimulation was applied after saccades. Instead, saccade facilitation can be regarded as memory effects in the saccadic system. Such effects appear to be equivalent to those induced by a positive reinforcement signal, or natural reward. When the monkey performed the biased-reward saccade task, a visually guided saccade task in which only one target position is associated with a large reward throughout a block, the reaction times of saccades toward the particular target associated with a large reward become shorter while the reaction times of saccades toward the target associated with a small or no reward become shorter (for review, see Hikosaka et al., 2006).

Similarities between the effects of electrical stimulation and natural reward were also evident when stimulation/reward position contingency was switched. In the biased-reward saccade task, the reward position contingency was constant within a block but was reversed between blocks with no instruction. When an unexpected delivery of reward was detected (i.e., at the transition from a small to large reward block), the saccade reaction time for that direction became shorter quickly; when an unexpected lack of reward was detected (i.e., at the transition from a large to small

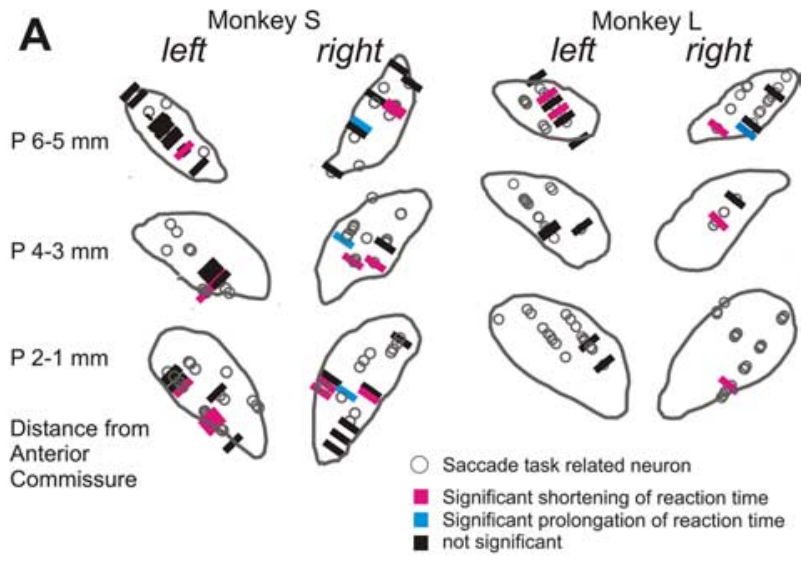

B

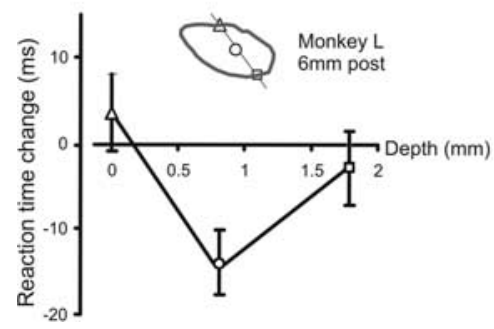

Figure 7. $\quad \boldsymbol{A}$, Recording and stimulation sites in monkey $S$ (left) and monkey $L$ (right) are projected onto coronal sections with $2 \mathrm{~mm}$ intervals traced from magnetic resonance images. The numbers on the left indicate distance (in millimeters) posterior to the anterior commissure. The open circles denote the locations of neurons that showed any type of task-related activity during the performance of the biased-reward saccade task (see Materials and Methods). The bars denote the locations of electrical stimulation. Reaction time changes of contraversive saccades by post-contraversive saccadic stimulation are color coded: pink, significant decrease; cyan, significant increase; black, no significant changes. $\boldsymbol{B}$, The effect of electrical stimulation was localized in the caudate. Stimulation was applied at three different depths in a single electrode track: upper border (triangle), central part (circle), and lower border (square) of the caudate. The mean and SE of the reaction time changes are shown for each depth from the upper border of the caudate. The results are based on one set of three stimulation experiments performed along the same penetration (monkey $\mathrm{L}, 6 \mathrm{~mm}$ posterior to the anterior commissure).

reward block), the saccade reaction time for that direction became longer, but slowly (Watanabe and Hikosaka, 2005). We found a similar asymmetry in the change of the saccade reaction time using the postsaccadic electrical stimulation in the caudate. 
Shortening of the saccade reaction time was quick after the stimulation started, whereas the saccade reaction time remained short even after the stimulation was stopped.

These results suggest that a common neural mechanism underlies the reaction time changes induced by natural reward and those induced by caudate stimulation. In the biased-reward saccade task, changes in caudate neuronal activity correlate with the changes in the saccade reaction times (Watanabe and Hikosaka, 2005). Therefore, the electrical stimulation in the caudate may induce similar changes in caudate neuronal activity, which then would induce asymmetric changes in saccade reaction times.

\section{Saccade direction specificity}

Electrical stimulation in the caudate could facilitate neuronal processes locally but nonspecifically, regardless of the direction of saccades associated with the stimulation. Thus, stimulation after ipsiversive saccades should still cause a shortening in the reaction time of contraversive saccades, because the activity of a majority of caudate projection neurons are contralateral-preferred (Hikosaka et al., 1989a,b). We found, instead, that the post-ipsiversive saccade stimulation caused a shortening in the reaction times of ipsiversive saccades. This result indicates that the effects of postsaccadic stimulation depend on the association of saccades to a particular direction and the subsequent electrical stimulation. This supports the hypothesis that the synaptic efficacy of corticostriatal projections that carry unique spatial and motor information is strengthened at the level of the striatum (Reynolds and Wickens, 2002).

A question remains as to the interchangeability of the caudate stimulation effects. Stimulation after contraversive saccades led to the shortening or prolongation of the reaction times of contralateral and ipsilateral saccades, respectively, whereas stimulation after ipsiversive saccades led to the shortening or prolongation of the reaction times of ipsilateral and contralateral saccades, respectively. This might suggest that the caudate has a potential to control saccades in both directions. It is noteworthy in this respect that the preferred direction of single caudate projection neurons could change flexibly depending on the direction of expected reward (Kawagoe et al., 1998; Ding and Hikosaka, 2006). Such flexible modulation may be supported by the bilateral corticostriatal connections (Carpenter, 1981; Royce, 1982). Either natural reward or caudate stimulation may be capable of guiding caudate projection neurons to be tuned to whichever direction or position that is associated with reward or stimulation.

It is worth noting that this saccade direction selectivity of stimulation effects may also depend on the task context or the stimulation sites. For example, Williams and Eskandar (2006) reported behavioral changes produced by electrical stimulation in the caudate. In this study, caudate stimulation promotes an association between visual stimulus, regardless of its location, and motor response, whereas in our study caudate stimulation promoted an association between visual stimulus location and motor response. The difference might also be attributable to the regional difference within the caudate. Williams and Eskandar (2006) focused on the head (anterior part) of the caudate, which receives projections from the frontal association cortex (Selemon and Goldman-Rakic, 1985; Yeterian and Pandya, 1991) including the dorsolateral prefrontal cortex, where different kinds of information are integrated for controlling purposeful behavior (Miller and Cohen, 2001). We focused on the body (more posterior part) of the caudate, which receives cortical inputs mainly from the oculomotor areas, especially the frontal eye field and the supplementary eye field (Shook et al., 1991; Parthasarathy et al., 1992) where the saccadic goal in space is determined (Bruce and Goldberg, 1985).

\section{Timing specificity}

Saccade direction selectivity and persistent effects, which were characteristics of the postsaccadic stimulation, were not observed with the presaccadic stimulation. The saccades that were associated with stimulation and were directed to the side of stimulation (ipsiversive saccades) were facilitated with the postsaccadic stimulation, but were delayed with the presaccadic stimulation. Unlike the postsaccadic stimulation, the presaccadic stimulation induced no persistent effect. These results suggest that the effect of the postsaccadic stimulation took the form of memory, whereas the presaccadic stimulation yielded direct and instantaneous motor effects.

Our result on the timing specific persistent effect is consistent with the finding by Williams and Eskandar (2006) showing that caudate stimulation was effective in improving learning of visual-motor associations when the stimulation was delivered after motor response, but not during the presentation of the visual stimulus. The cellular mechanisms that support this timing specificity remained to be clarified. It is possible that the temporal contingency between cortical stimulation and activity of striatal output neurons may affect the direction of the change in the membrane potential, LTP and LTD (Fino et al., 2005).

\section{Mechanisms of the effect of postsaccadic stimulation}

An important question is where and how electrical stimulation exerts its action to induce lasting changes in saccade reaction time. One plausible possibility is that plastic changes in neuronal processing occur in the caudate (Major and White, 1978). The locally modifiable elements may include axon terminals of extrinsic origin (dopaminergic terminals from the substantia nigra pars compacta and glutamatergic terminals from the cerebral cortex) and neurons in the caudate, namely striatal projection neurons or interneurons. Similarities in the effects of electrical stimulation and natural reward could indicate common mechanisms, namely release of dopamine. Involvement of dopamine in the change of the corticostriatal synaptic efficacy has been reported (Phillips et al., 1979; Wickens et al., 1996; Canales et al., 2002; Reynolds and Wickens, 2002). In support of this dopamine hypothesis is our previous finding that the reward-dependent modulation of the saccadic reaction times are attenuated after an injection of a dopamine $\mathrm{D}_{1}$ antagonist in the oculomotor region of caudate and was enhanced after an injection of a dopamine $\mathrm{D}_{2}$ antagonist (Nakamura and Hikosaka, 2006).

However, direct stimulation of dopaminergic terminal fibers is unlikely for the changes in saccade reaction times, because those axons are fine and are likely to have high activation thresholds. Previous studies have shown that the effect of a combination of presynaptic and postsynaptic activity, in addition to changes in dopamine release, induces LTD or LTP (for review, see Reynolds and Wickens, 2002). In our experiments, electrical stimulation in the caudate may depolarize the caudate projection neurons and/or stimulate glutamatergic corticostriatal terminals. If these events are combined with an increase in dopamine release caused by task-related activation of dopamine neurons (Kawagoe et al., 2004), caudate neurons may undergo facilitatory plastic changes.

Another possibility is that the observed effects of caudate stimulation are attributable to transsynaptic effects. The stimulation may affect dopamine neurons in the substantia nigra pars compacta directly (Gerfen, 1985) or indirectly through the substantia nigra pars reticulata (Tepper et al., 1995), subthalamic 
nucleus (Parent and Smith, 1987; Iribe et al., 1999), or globus pallidus (Bevan et al., 1996; Paladini et al., 1999). This line of thought is consistent with the induction of LTP by simultaneous activation of dopamine neurons and caudate projection neurons (Reynolds et al., 2001). Alternatively, electrical stimulation of the caudate, which has direct connection to the substantia nigra pars reticulata (Tulloch et al., 1978; Hedreen and DeLong, 1991; Parent and Hazrati, 1994), might induce a plastic change in GABA receptor expression in pars reticulata neurons (Katz et al., 2005). The outcome by this process could be robust because the substantia nigra pars reticulata is a major output of the basal ganglia for oculomotor control (Hikosaka et al., 2000). Yet another possibility is that caudate stimulation might activate cortical neurons antidromically (Selemon and Goldman-Rakic, 1985; Yeterian and Pandya, 1991; Parthasarathy et al., 1992), which then induce plastic effects inside the cortical area such as the frontal eye field.

Our recent studies, however, support the first hypothesis that a plastic change occurs, at least partly, within the caudate. The changes in saccade reaction times in the biased saccade task correlate with changes in caudate neuronal activity, especially at the transition between large and small reward blocks (Watanabe et al., 2003a). The reward-dependent modulation of the saccadic reaction times are attenuated after an injection of a dopamine $D_{1}$ antagonist in the oculomotor region of caudate and was enhanced after an injection of a dopamine $\mathrm{D}_{2}$ antagonist (Nakamura and Hikosaka, 2006).

In conclusion, the present results suggest that the electrical stimulation in the caudate induces behavioral changes comparable with those induced by natural reward. This supports the hypothesis that the linkage of sensorimotor and reward signals takes place, at least partly, in the caudate. We further found that the behavioral changes are induced in spatially and temporally specific manner in the caudate, by taking advantage of electrical stimulation, which allowed us to access to the reward mechanism directly.

\section{References}

Barnes TD, Kubota Y, Hu D, Jin DZ, Graybiel AM (2005) Activity of striatal neurons reflects dynamic encoding and recoding of procedural memories. Nature 437:1158-1161.

Bevan MD, Smith AD, Bolam JP (1996) The substantia nigra as a site of synaptic integration of functionally diverse information arising from the ventral pallidum and the globus pallidus in the rat. Neuroscience 75:5-12.

Bruce CJ, Goldberg ME (1985) Primate frontal eye fields. I. Single neurons discharging before saccades. J Neurophysiol 53:603-635.

Bursten B, Delgado JM (1958) Positive reinforcement induced by intracerebral stimulation in the monkey. J Comp Physiol Psychol 51:6-10.

Calabresi P, Pisani A, Mercuri NB, Bernardi G (1996) The corticostriatal projection: from synaptic plasticity to dysfunctions of the basal ganglia. Trends Neurosci 19:19-24.

Canales JJ, Capper-Loup C, Hu D, Choe ES, Upadhyay U, Graybiel AM (2002) Shifts in striatal responsivity evoked by chronic stimulation of dopamine and glutamate systems. Brain 125:2353-2363.

Carpenter MB (1981) Anatomy of the corpus striatum and brain stem integrating systems. In: The nervous system (Brooks VB, ed), pp 947-995. Bethesda, MD: American Physiological Society.

Charpier S, Deniau JM (1997) In vivo activity-dependent plasticity at cortico-striatal connections: evidence for physiological long-term potentiation. Proc Natl Acad Sci USA 94:7036-7040.

Charpier S, Mahon S, Deniau JM (1999) In vivo induction of striatal longterm potentiation by low-frequency stimulation of the cerebral cortex. Neuroscience 91:1209-1222.

Ding L, Hikosaka O (2006) Comparison of reward modulation in the frontal eye field and caudate of the macaque. J Neurosci 26:6695-6703.

Everitt BJ, Robbins TW (2005) Neural systems of reinforcement for drug addiction: from actions to habits to compulsion. Nat Neurosci 8:1481-1489.
Fino E, Glowinski J, Venance L (2005) Bidirectional activity-dependent plasticity at corticostriatal synapses. J Neurosci 25:11279-11287.

Gerfen CR (1985) The neostriatal mosaic. I. Compartmental organization of projections from the striatum to the substantia nigra in the rat. J Comp Neurol 236:454-476.

Haruno M, Kawato M (2006) Different neural correlates of reward expectation and reward expectation error in the putamen and caudate nucleus during stimulus-action-reward association learning. J Neurophysiol 95:948-959.

Haruno M, Kuroda T, Doya K, Toyama K, Kimura M, Samejima K, Imamizu H, Kawato M (2004) A neural correlate of reward-based behavioral learning in caudate nucleus: a functional magnetic resonance imaging study of a stochastic decision task. J Neurosci 24:1660-1665.

Hassani OK, Cromwell HC, Schultz W (2001) Influence of expectation of different rewards on behavior-related neuronal activity in the striatum. J Neurophysiol 85:2477-2489.

Hedreen JC, DeLong MR (1991) Organization of striatopallidal, striatonigral, and nigrostriatal projections in the macaque. J Comp Neurol 304:569-595.

Hikosaka O, Sakamoto M, Usui S (1989a) Functional properties of monkey caudate neurons. I. Activities related to saccadic eye movements. J Neurophysiol 61:780-798.

Hikosaka O, Sakamoto M, Usui S (1989b) Functional properties of monkey caudate neurons. II. Visual and auditory responses. J Neurophysiol 61:799-813.

Hikosaka O, Sakamoto M, Usui S (1989c) Functional properties of monkey caudate neurons. III. Activities related to expectation of target and reward. J Neurophysiol 61:814-832.

Hikosaka O, Sakamoto M, Miyashita N (1993) Effects of caudate nucleus stimulation on substantia nigra cell activity in monkey. Exp Brain Res 95:457-472.

Hikosaka O, Takikawa Y, Kawagoe R (2000) Role of the basal ganglia in the control of purposive saccadic eye movements. Physiol Rev 80:953-978.

Hikosaka O, Nakamura K, Nakahara H (2006) Basal ganglia orient eyes to reward. J Neurophysiol 95:567-584.

Iribe Y, Moore K, Pang KC, Tepper JM (1999) Subthalamic stimulationinduced synaptic responses in substantia nigra pars compacta dopaminergic neurons in vitro. J Neurophysiol 82:925-933.

Jog MS, Kubota Y, Connolly CI, Hillegaart V, Graybiel AM (1999) Building neural representations of habits. Science 286:1745-1749.

Justesen DR, Sharp JC, Porter PB (1963) Self-stimulation of the caudate nucleus by instrumentally naive cats. J Comp Physiol Psychol 56:371-374.

Katz J, Nielsen KM, Soghomonian JJ (2005) Comparative effects of acute or chronic administration of levodopa to 6-hydroxydopamine-lesioned rats on the expression of glutamic acid decarboxylase in the neostriatum and GABAA receptors subunits in the substantia nigra, pars reticulata. Neuroscience 132:833-842.

Kawagoe R, Takikawa Y, Hikosaka O (1998) Expectation of reward modulates cognitive signals in the basal ganglia. Nat Neurosci 1:411-416.

Kawagoe R, Takikawa Y, Hikosaka O (2004) Reward-predicting activity of dopamine and caudate neurons-a possible mechanism of motivational control of saccadic eye movement. J Neurophysiol 91:1013-1024.

Kitama T, Ohno T, Tanaka M, Tsubokawa H, Yoshida K (1991) Stimulation of the caudate nucleus induces contraversive saccadic eye movements as well as head turning in the cat. Neurosci Res 12:287-292.

Lauwereyns J, Takikawa Y, Kawagoe R, Kobayashi S, Koizumi M, Coe B, Sakagami M, Hikosaka O (2002a) Feature-based anticipation of cues that predict reward in monkey caudate nucleus. Neuron 33:463-473.

Lauwereyns J, Watanabe K, Coe B, Hikosaka O (2002b) A neural correlate of response bias in monkey caudate nucleus. Nature 418:413-417.

Lehericy S, Benali H, Van de Moortele PF, Pelegrini-Issac M, Waechter T, Ugurbil K, Doyon J (2005) Distinct basal ganglia territories are engaged in early and advanced motor sequence learning. Proc Natl Acad Sci USA 102:12566-12571.

Levine MS, Ferguson N, Kreinick CJ, Gustafson JW, Schwartzbaum JS (1971) Sensorimotor dysfunctions and aphagia and adipsia following pallidal lesions in rats. J Comp Physiol Psychol 77:282-293.

Lovinger DM, Partridge JG, Tang KC (2003) Plastic control of striatal glutamatergic transmission by ensemble actions of several neurotransmitters and targets for drugs of abuse. Ann NY Acad Sci 1003:226-240.

Mahon S, Deniau JM, Charpier S (2004) Corticostriatal plasticity: life after the depression. Trends Neurosci 27:460-467. 
Major R, White N (1978) Memory facilitation by self-stimulation reinforcement mediated by the nigro-neostriatal bundle. Physiol Behav 20:723-733.

Miller EK, Cohen JD (2001) An integrative theory of prefrontal cortex function. Annu Rev Neurosci 24:167-202.

Miyachi S, Hikosaka O, Lu X (2002) Differential activation of monkey striatal neurons in the early and late stages of procedural learning. Exp Brain Res 146:122-126.

Nakamura K, Hikosaka O (2006) Role of dopamine in the primate caudate nucleus in reward modulation of saccades. J Neurosci 26:5360-5369.

O’Doherty J, Dayan P, Schultz J, Deichmann R, Friston K, Dolan RJ (2004) Dissociable roles of ventral and dorsal striatum in instrumental conditioning. Science 304:452-454.

Olds J, Milner P (1954) Positive reinforcement produced by electrical stimulation of septal area and other regions of rat brain. J Comp Physiol Psychol 47:419-427.

Olds ME, Fobes JL (1981) The central basis of motivation: intracranial selfstimulation studies. Annu Rev Psychol 32:523-574.

Packard MG, Knowlton BJ (2002) Learning and memory functions of the basal ganglia. Annu Rev Neurosci 25:563-593.

Paladini CA, Celada P, Tepper JM (1999) Striatal, pallidal, and pars reticulata evoked inhibition of nigrostriatal dopaminergic neurons is mediated by GABA(A) receptors in vivo. Neuroscience 89:799-812.

Parent A, Hazrati LN (1994) Multiple striatal representation in primate substantia nigra. J Comp Neurol 344:305-320.

Parent A, Smith Y (1987) Organization of efferent projections of the subthalamic nucleus in the squirrel monkey as revealed by retrograde labeling methods. Brain Res 436:296-310.

Parthasarathy HB, Schall JD, Graybiel AM (1992) Distributed but convergent ordering of corticostriatal projections: analysis of the frontal eye field and the supplementary eye field in the macaque monkey. J Neurosci 12:4468-4488

Partridge JG, Tang KC, Lovinger DM (2000) Regional and postnatal heterogeneity of activity-dependent long-term changes in synaptic efficacy in the dorsal striatum. J Neurophysiol 84:1422-1429.

Phillips AG, Carter DA, Fibiger HC (1976) Dopaminergic substrates of intracranial self-stimulation in the caudate-putamen. Brain Res 104:221-232.

Phillips AG, Mora F, Rolls ET (1979) Intracranial self-stimulation in orbitofrontal cortex and caudate nucleus of rhesus monkey: effects of apomorphine, pimozide, and spiroperidol. Psychopharmacology (Berl) 62:79-82.

Plotnik RD, Mir D, Delgado MR (1972) Map of reinforcing sites in the rhesus monkey brain. Int J Psychobiol 2:1-21.

Reynolds JN, Wickens JR (2000) Substantia nigra dopamine regulates synaptic plasticity and membrane potential fluctuations in the rat neostriatum, in vivo. Neuroscience 99:199-203.

Reynolds JN, Wickens JR (2002) Dopamine-dependent plasticity of corticostriatal synapses. Neural Netw 15:507-521.
Reynolds JN, Hyland BI, Wickens JR (2001) A cellular mechanism of reward-related learning. Nature 413:67-70.

Royce GJ (1982) Laminar origin of cortical neurons which project upon the caudate nucleus: a horseradish peroxidase investigation in the cat. J Comp Neurol 205:8-29.

Schultz W, Tremblay L, Hollerman JR (2003) Changes in behavior-related neuronal activity in the striatum during learning. Trends Neurosci 26:321-328.

Selemon LD, Goldman-Rakic PS (1985) Longitudinal topography and interdigitation of corticostriatal projections in the rhesus monkey. J Neurosci 5:776-794.

Shook BL, Schlag-Rey M, Schlag J (1991) Primate supplementary eye field. II. Comparative aspects of connections with the thalamus, corpus striatum, and related forebrain nuclei. J Comp Neurol 307:562-583.

Takikawa Y, Kawagoe R, Hikosaka O (2002) Reward-dependent spatial selectivity of anticipatory activity in monkey caudate neurons. J Neurophysiol 87:508-515.

Tepper JM, Martin LP, Anderson DR (1995) $\mathrm{GABA}_{\mathrm{A}}$ receptor-mediated inhibition of rat substantia nigra dopaminergic neurons by pars reticulata projection neurons. J Neurosci 15:3092-3103.

Tulloch IF, Arbuthnott GW, Wright AK (1978) Topographical organization of the striatonigral pathway revealed by anterograde and retrograde neuroanatomical tracing techniques. J Anat 127:425-441.

Watanabe K, Hikosaka O (2005) Immediate changes in anticipatory activity of caudate neurons associated with reversal of position-reward contingency. J Neurophysiol 94:1879-1887.

Watanabe K, Lauwereyns J, Hikosaka O (2003a) Effects of motivational conflicts on visually elicited saccades in monkeys. Exp Brain Res 152:361-367.

Watanabe K, Lauwereyns J, Hikosaka O (2003b) Neural correlates of rewarded and unrewarded eye movements in the primate caudate nucleus. J Neurosci 23:10052-10057.

White NM, Hiroi N (1998) Preferential localization of self-stimulation sites in striosomes/patches in the rat striatum. Proc Natl Acad Sci USA 95:6486-6491.

White NM, McDonald RJ (2002) Multiple parallel memory systems in the brain of the rat. Neurobiol Learn Mem 77:125-184.

Wickens JR, Begg AJ, Arbuthnott GW (1996) Dopamine reverses the depression of rat corticostriatal synapses which normally follows highfrequency stimulation of cortex in vitro. Neuroscience 70:1-5.

Williams ZM, Eskandar EN (2006) Selective enhancement of associative learning by microstimulation of the anterior caudate. Nat Neurosci 9:562-568.

Wurtz RH, Olds J (1963) Amygdaloid stimulation and operant reinforcement in the rat. J Comp Physiol Psychol 56:941-949.

Yeterian EH, Pandya DN (1991) Prefrontostriatal connections in relation to cortical architectonic organization in rhesus monkeys. J Comp Neurol 312:43-67. 